\title{
Microplate Fixation without Maxillomandibular Fixation in Double Mandibular Fractures
}

\author{
Seung Wook Song, \\ Jin Sik Burm, \\ Won Yong Yang, \\ Sang Yoon Kang \\ Department of Plastic and Reconstructive \\ Surgery, Kyung Hee University School of \\ Medicine, Seoul, Korea
}

No potential conflict of interest relevant to this article was reported.

\begin{abstract}
Background: Maxillomandibular fixation (MMF) is usually used to treat double mandibular fractures. However, advancements in reduction and fixation techniques may allow recovery of the premorbid dental arch and occlusion without the use of MMF. We investigated whether anatomical reduction and microplate fixation without MMF could provide secure immobilization and correct occlusion in double mandibular fractures. Methods: Thirty-four patients with double mandibular fractures were treated with open reduction and internal fixation without MMF. Both fracture sites were surgically treated. For bony fixations, we used microplates with or without wire. After reduction, each fracture site was fixed at two or three points to maintain anatomical alignment of the mandible. Interdental wiring was used to reduce the fracture at the superior border and to enhance stability for 6 weeks. Mouth opening was permitted immediately.

Results: No major complications were observed, including infection, plate exposure, non-union, or significant malocclusion. Five patients experienced minor complications, among whom the only one patient experienced a persistant but mild malocclusion with no need for additional management.

Conclusion: This study showed that double mandibular fractures correction with twoor three-point fixation without MMF simplified the surgical procedure, increased patient comfort, and reduced complications, due to good stability and excellent adaptation.
\end{abstract}

Keywords: Mandibular fractures / Jaw fixation techniques / External fixators

\section{INTRODUCTION}

The surgical principle underlying treatment of mandibular fractures is to restore occlusion to the premorbid state through accurate reduction and proper fixation. Perioperative maxillomandibular fixation (MMF) is commonly used to aid in the reduction, stabilization, and fixation of mandibular fractures, particularly for double or comminuted fractures because malocclusion is a worrisome entity without MMF. Recently, advancements in oper-

\footnotetext{
Correspondence: Jin Sik Burm

Department of Plastic and Reconstructive Surgery, Kyung Hee University Medical Center, Kyung Hee University School of Medicine, 23 Kyungheedae-ro, Dongdaemun-gu, Seoul 130-872, Korea E-mail: jsburm@gmail.com

*This article was presented at the 3rd Research and Reconstructive Forum on May 9-10, 2013.
}

Received May 24, 2014 / Revised July 25, 2014 / Accepted August 1, 2014 ative techniques have allowed clinicians to achieve intraoperative anatomical reduction and stable fixation of the mandible to restore premorbid occlusion without requiring MMF in isolated unilateral fractures [1-4]. If the fractured mandible could be precisely reduced and fixed in its original position, the mandible may be able to recover its premorbid dental arch and occlusion without MMF, even in double fractures.

The purpose of this article is to introduce the feasibility and technical details of anatomical reduction and microplate fixation without MMF in reconstruction of double mandibular fractures.

\section{METHODS}

Thirty-four patients (27 men, 7 women; age range, 14 to 65 years; mean, 30.3 years) with double mandibular fractures were treated 
with MMF-less open reduction and internal fixation. All patients received surgical treatment at both mandibular fracture sites. The distribution of fracture sites is shown in Table 1. All of the patients included in the study had double mandubular fractures at two different sites along the mandible. We included the double fractures of the same side in one case (left condyle and left parasymphysis). Fractures involving three fracture sites were not included in this series. Parasymphyseal fractures were classified as symphyseal fractures, and subcondyle and condylar neck fractures were classified as condylar fractures. Condylar head fractures and severe comminuted fractures were not included in this study.

Interdental wiring was applied preoperatively on each fracture line with or without a commercial mandibular bandage. Manual reduction was the initial treatment method. The interval between injury and operation was 4 to 12 days (average of 6.1 days). All pa-

Table 1. Distribution of fracture sites in 34 patients with a double mandibular fractures

\begin{tabular}{lc}
\hline Fracture site & No. of patients \\
\hline Symphysis $^{\mathrm{a})}$ and condyle & 18 \\
\hline Symphysis and angle & 10 \\
\hline Bilateral condyle & 3 \\
Symphysis and ramus & 2 \\
\hline Body and condyle & 1 \\
\hline Total & 34 \\
\hline
\end{tabular}

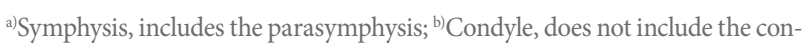
dylar head.

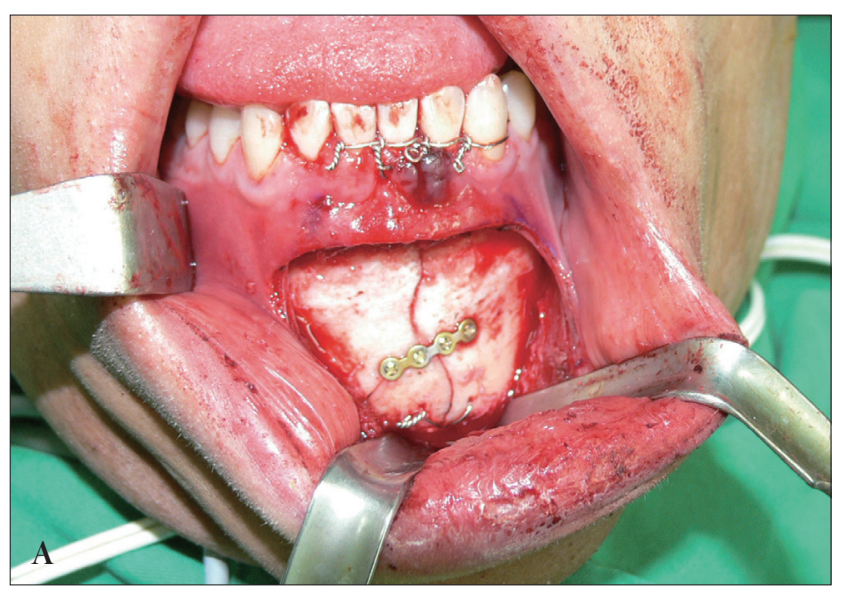

tients were assessed both clinically and radiographically during the follow-up period, ranging from 3 to 29 months. We assessed alignment of the fracture, tested occlusion status (thin paper bite test and asking the patients about the discomfort and difference from premorbid occlusion during biting), and addressed complications during this follow-up period.

Fractures of the symphyseal and body regions of the mandible were exposed through an incision through the labial mucosa, placed 6-7 mm above the inferior buccal sulcus (Fig. 1A). Subperiosteal dissection was extended from the incision down over the mandibular margin and under to the inferior portion of the inner cortex. Any laceration wound in the submandibular area was used to expose the fractures. In angle fractures, the superior border of the fracture was intraorally exposed with an incision, approximately $2 \mathrm{~cm}$ long, placed posterior to the third molar and lateral to the anterior border of the coronoid process. The third molar in the fracture line was typically extracted prior to fracture reduction. The condyle and the inferior border of the angle were exposed through an external approach (Fig. 1B).

The initial interdental wiring was removed before bone reduction. All granulated tissues and/or calluses in the fracture lines were removed. Reduction and fixation of the fracture was achieved with manual manipulation and bone-reduction forceps. In general, a two- or three-point fixation technique was performed at each fracture site with microplates, with or without

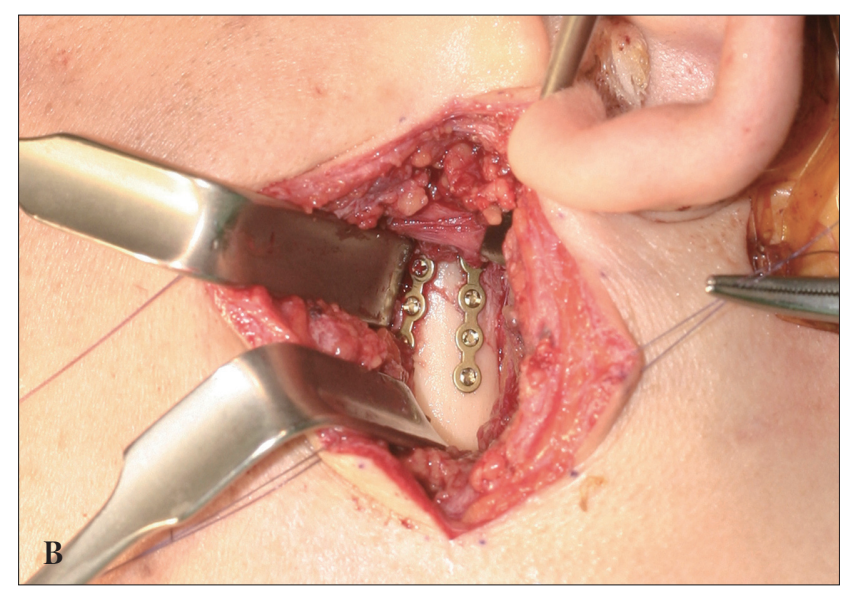

Fig. 1. Mandibular fracture fixations without maxillomandibular fixation. (A) Three-point fixation was utilized for symphyseal fractures. Fixation points were at the inferior border, superior border (interdental wiring), and middle of the outer cortex in that order. (B) Two-point fixation was employed for condyle fractures. Fixation points were at the posterior border and the middle of the outer cortex. 
wire. Most microplates were fixed with $1.2-\mathrm{mm}$ monocortical screws, 3- to 4-mm in length (Stryker Leibinger, Freiburg, Germany). Wire fixation was frequently used at the inferior border when reduction was not well maintained with manipulation or bone-reduction forceps. After two holes were made with a drill to the inner cortex, the wire was inserted through the holes and tightened. In symphyseal and body fractures, fixation was applied at the inferior border, at the superior border (with interdental wiring), and in the middle of the fracture line, in that order (Fig. 1A). Fixation at the inferior border was confirmed by the absence of vertical misalignment and no fracture gap along the mandibular margin. New interdental wiring was applied to four teeth, two on each side of the fracture line, to accurately align the fracture edges of the inner and outer cortex at the superior border. Once an adequate dental arch and occlusion were obtained, the middle of the fracture line on the outer cortex was fixed with a plate system. The plate was typically placed perpendicular to the fracture line. Angle fractures were typically fixed at the superior and inferior borders. Condylar fractures were fixed at the posterior border and the outer cortex (Fig. 1B). These fixation sites were selected to ensure that the bony edges of the fracture at the lingual, inner cortices would be adjoined tightly. After internal fixation, dental occlusion was evaluated by a thin paper bite test ( $0.1 \mathrm{~mm}$ thick) on both sides of molars and premolars and by visual inspection during passive opening and closing the mouth. If proper occlusion was not achieved, the fracture reductions were readjusted.

After primary closure of incisions, a mild compressive dressing was applied over the areas of subperiosteal dissection. For chin support, an elastic facial bandage was applied to limit excessive mouth opening for seven days postoperatively. Interdental wiring was maintained for six weeks. Mouth opening was permitted immediately. Patients were provided a liquid diet for the first three or four days after the operation, and a soft diet for the next six weeks. Oral rinsing was recommended before resuming gentle tooth brushing on the third day after surgery.

\section{RESULTS}

All fractures exhibited excellent bone healing without major complications. We did not observe wound dehiscence, infection, significant malocclusion, or temporomandibular joint problems, which might have required further treatment during the followup period (Figs. 2, 3). Radiographs taken at the last follow-up visit revealed complete bony consolidation of fracture lines without evidence of malunion, nonunion, or partial union. Three months after surgery, the mean inter-incisor distance of full mouth opening was $44.1 \mathrm{~mm}$, ranging from 36 to $52 \mathrm{~mm}$.

Five patients experienced minor complications. These included a minor degree of malocclusion without functional limitations, an asymptomatic microplate fracture, and delayed bony union (Table 2). Malocclusion was revealed by the thin paper bite test, and the patient had reported occlusal discomfort in the early postoperative period (seven days). Mild malocclusion occurred in two patients with double fractures involving the parasymphysis. These were fixed with microplates at the middle and inferior portions of the anterior cortex, rather than at the inferior margin of the mandible. One patient had no occlusal discomfort six months postoperatively, despite signs of a minimal, unilateral open bite in the molar region. Another patient demonstrated minimal premature occlusal contact, with signs of minimal open bite at the 11month follow-up visit, but required no additional treatment. An asymptomatic microplate fracture was observed in one of two microplates placed on a left subcondyle fracture in a patient with right condylar neck and left subcondyle fractures. This was an incidental observation from a regular follow-up X-ray at five months, and the bony union process had already completed. An asymptomatic delayed union was observed in a symphyseal fracture in a patient with symphyseal and right subcondyle fractures six months postoperation. At 12 months, complete union was observed. The single patient with a condylar neck fracture had exhibited temporary palsy of the frontal branch of facial nerve. That patient had fully recovered three months later.

In our series, there was no major complication. Minor symptomatic complications directly related to microplate fixation without MMF were identifiable in two patients $(6 \%)$ who had complained of minimal malocclusion in the early follow-up period and in one patient (3\%) at the last follow-up visit with no need for additional treatment. 

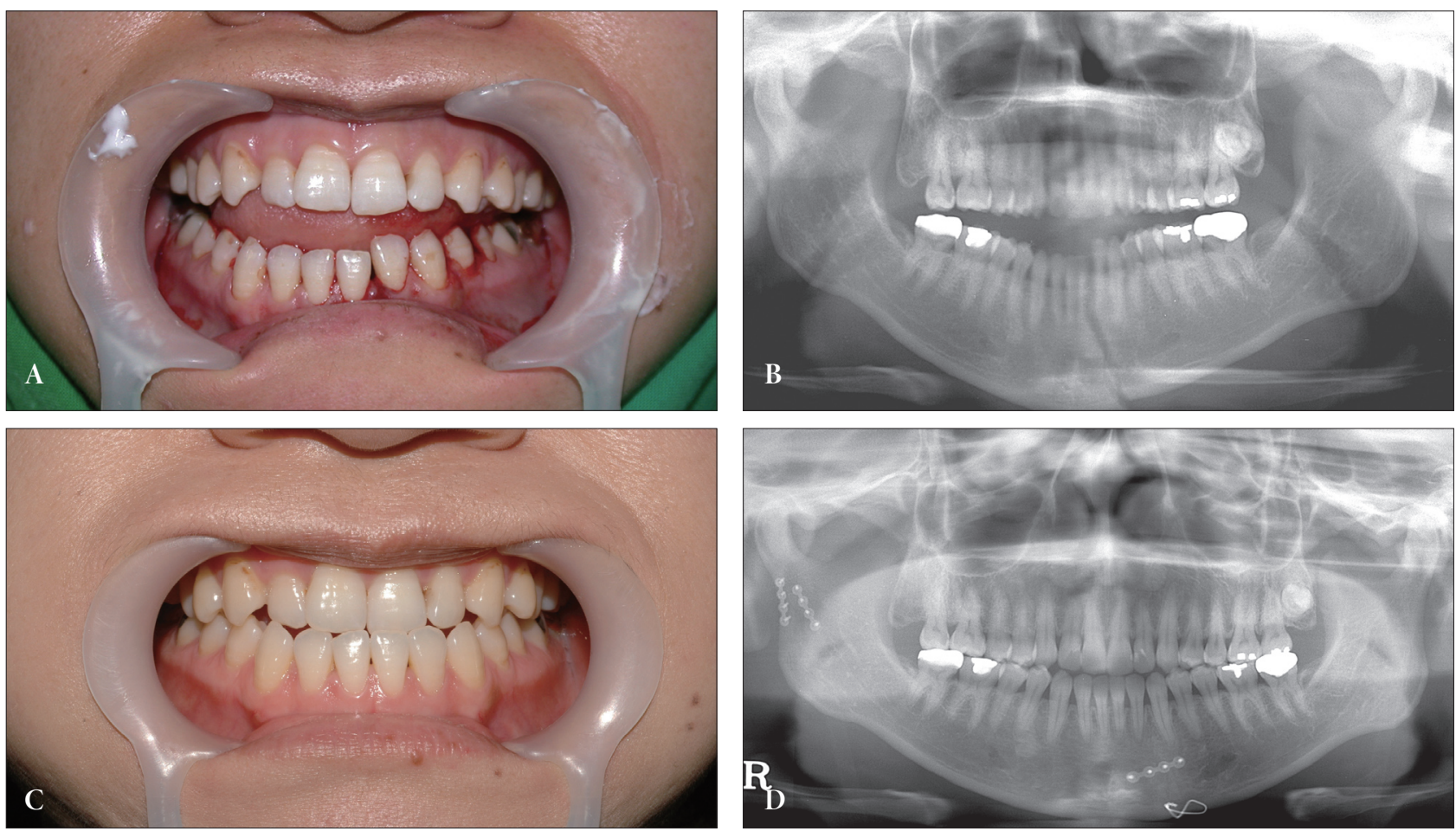

Fig. 2. Double fractures at symphysis and right subcondyle. (A, B) Preoperative occlusal photograph and panoramic radiograph. (C, D) Complete occlusal restoration and bony consolidation was observed 12 months postoperatively, despite premorbid type III occlusion.
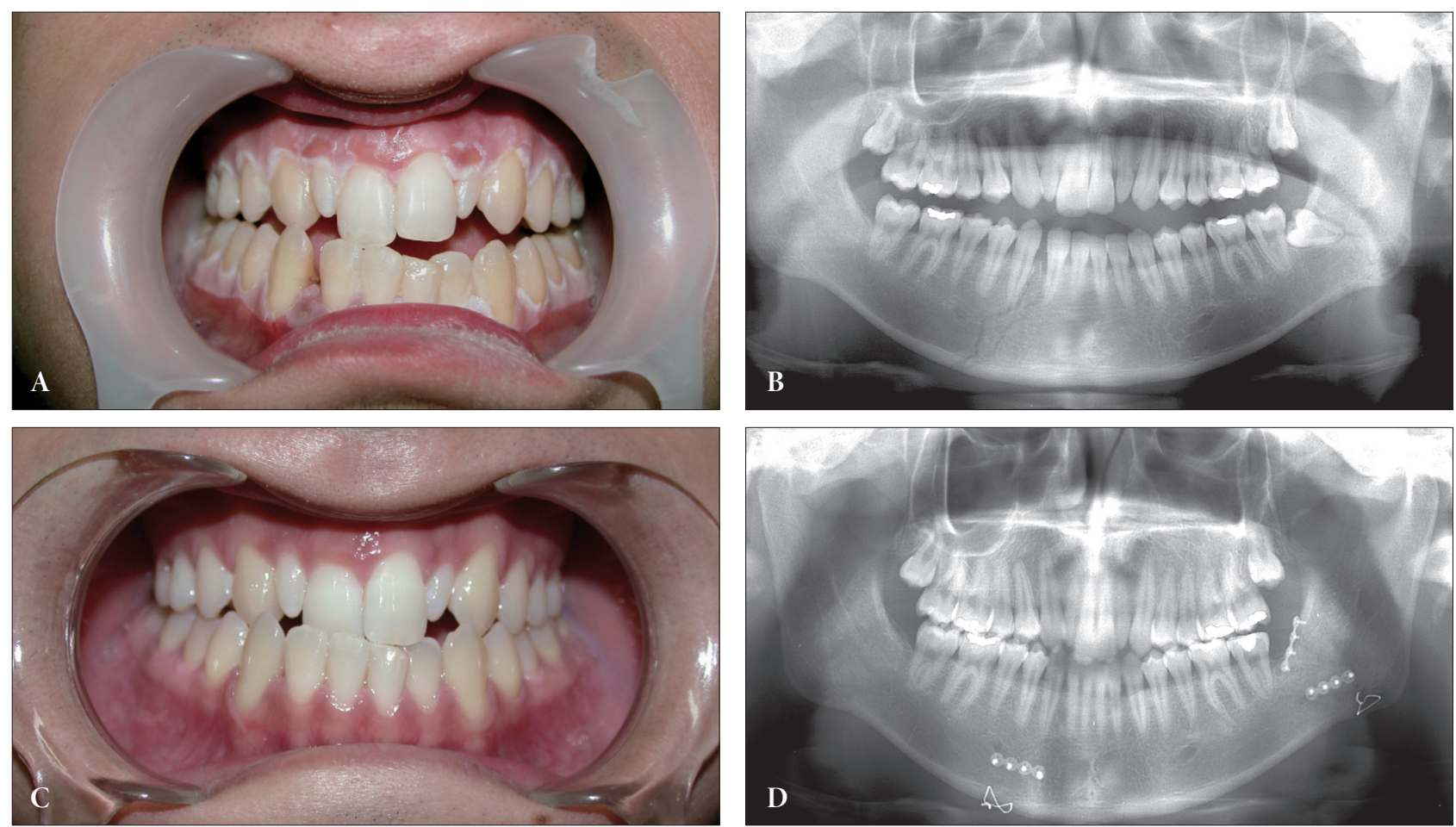

Fig. 3. Double fractures at symphysis and left angle. (A, B) Preoperative occlusal photograph and panoramic radiograph. (C, D) Excellent occlusion and complete bone healing was observed four months postoperatively, despite premorbid type III occlusion. 
Table 2. Complications after reconstruction without maxillomandibular fixation occurred in 5 of 34 patients with double mandibular fractures

\begin{tabular}{|c|c|c|c|c|c|c|c|}
\hline No. & Age/Sex & Fracture site & Cause & Fixation & Complication & Residual complication & Follow-up (mo) \\
\hline 1 & $22 / \mathrm{M}$ & $\begin{array}{l}\text { Rt. parasymphysis } \\
\text { Lt. subcondyle }\end{array}$ & Assault & $\mathrm{P}, \mathrm{P}, \mathrm{IW} / \mathrm{P}, \mathrm{P}$ & Mild malocclusion & - & 6 \\
\hline 2 & $30 / \mathrm{M}$ & $\begin{array}{l}\text { Rt. parasymphysis } \\
\text { Lt. angle }\end{array}$ & Assault & $\begin{array}{l}\text { P, P, IWW, } \\
\text { P, P }\end{array}$ & Mild malocclusion & + & 11 \\
\hline 3 & $32 / \mathrm{M}$ & $\begin{array}{l}\text { Rt. condylar neck } \\
\text { Lt. subcondyle }\end{array}$ & Fall & $\mathrm{P}, \mathrm{P} / \mathrm{P}, \mathrm{P}$ & Asymptomatic microplate fracture & + & 5 \\
\hline 4 & $35 / F$ & $\begin{array}{l}\text { Symphysis } \\
\text { Rt.subcondyle }\end{array}$ & Fall & W, P, IW/P, P & Asymptomatic delayed union & - & 12 \\
\hline 5 & $56 / \mathrm{M}$ & $\begin{array}{l}\text { Symphysis } \\
\text { Lt. condylar neck }\end{array}$ & Blow & P, P, IWN, P & Frontal nerve palsy & - & 4 \\
\hline
\end{tabular}

M, male; Rt., right; Lt., left; P, plate; IW, interdental wiring; W, wiring; F, female.

\section{DISCUSSION}

MMF with arch bars has been used perioperatively to aid in mandibular fracture reduction and stabilization, fixation maintenance, and/or occlusal establishment. This approach may extend the operation time and can result in discomfort or poor oral hygiene for the patient due to limitations to mouth opening. MMF with intermaxillary fixation screws overcome the discomfort of MMF with arch bars, however there is still the possibility of teeth root injury and technical errors. In addition, MMF with screws has only a vertical vector but not a horizontal vector because screws must be positioned superior to the maxillary tooth roots and inferior to the mandibular tooth roots, while interdental wiring has a horizontal vector. Therefore, MMF may not exert a reduction force along the superior border of the fracture line.The traction force of MMF acts vertically only on the buccal aspect of the mandible and can induce an inward tilt of the teeth or an upward splay of the inferior border. This may result in rotation of bony segments and incomplete occlusal contact, especially in double or comminuted fractures.

Fordyce et al. [4] described several situations where a perioperative MMF was indicated. These included an operator or supervising consultant that preferred fracture reduction with MMF; the lack of an adequately trained assistant during the operation; the presence of a unilateral or bilateral condylar fracture that may require postoperative MMF; a planned postoperative MMF to immobilize the mandible and protect the fixation due to poor-quality internal fixation; an established infection; or a lack of patient compliance in the postoperative care of fracture sites. Many of these indications were related to the degree of surgical skill in reduction and fixation. However, when the fractured mandible can be accurately fixed under reduction in its original position, immediate re-establishment of the premorbid mandibular dental arch and occlusion may be achieved without MMF.

A cross-section of the mandible looks like a long curved trapezoid or triangle with round edges. The ideal method for achieving the original dental arch of the premorbid mandible may be to reduce and fix all fracture lines through the four sides, which are the superior margin, the inferior margin, and the inner and outer cortices. However, directly fixing the fracture through the inner cortex is very difficult. In a double fracture, fixation of the outer cortex alone could provide normal alignment, but may also result in vertical misalignment or rotation of the bone segments or dental arch widening due to the wide fracture gap of the inner cortex with subsequent malocclusion. However, fixation of both the superior and inferior borders of the mandible could simulataneously reduce the fractures along the inner and outer cortices. In our series, three-point fixation of the inferior border, the superior border (as interdental wiring) and the middle was performed for symphyseal or body fractures; two-point fixation of the superior and inferior borders was performed for angle fractures; and, twopoint fixation of the posterior border and the outer cortex was performed for condylar fractures.

Interdental wiring was performed as external fixation of the superior border of the symphysis and body. This is different from internal fixation of the superior border of the mandible described 
by other surgeons, which may be comparable to our internal fixation of the middle of the mandible. The reduction force of interdental wiring works along the superior borders of both the outer and inner cortices. This results in simultaneous reduction of the fracture gap at the inner and outer cortices. Interdental wiring is maintained for six weeks until sufficiant bony consolidation of the mandible is established. Interdental wiring also allows for immediate mouth opening, providing an excellent level of comfort and accessibility for patients to eat or drink and brush their teeth, and is readily removed with minimal pain. In our experience, the simple circumferential type was easier to apply and was more effective than the eyelet type. However, interdetal wiring is only useful when teeth around the fracture are intact. In the presence of teeth loss, we applied a microplate at a superior ridge of the fracture for a fixation.

As the materials of internal fixation, microplates or wires are less rigid than miniplates. Nevertheless, since it is always recommended that patients avoid active mastication and hard food for six weeks postoperatively and to minimize masticatory forces across the fracture sites for the first few months after injury [5], structural stability of the fracture site may be effectively maintained. The main purpose of microplate fixation is not a rigid fixation of fracture lines, but a fixation at the multiple essential points to maintain the anatomical reduction. The wire fixation used at the inferior border is not only used as a reduction tool but also internal fixator. Because it tightens the inner cortex and outer cortex simultaneously, the wire can provide more bony contact and maintain reduction. In our series, complications were represented by a minor degree of malocclusion, which gradually improved through adaptation and self-adjustment. We had one case of asymptomatic microplate fracture on the subcondyle, which did not prevent complete bone consolidation. At the last follow-up, residual symptomatic complication was apparent in only a single case with mild malocclusion and no need for additional treatment (3\%). In all cases, there was no wound dehiscence or infection due to the small dissection site and minimal mass effect. Therefore, we think that if anatomical reduction is adequately achieved, microplates can be applied to most mandibular fractures. In fact, this microplate fixation system has been used even in maxillary and mandibular complex fracture, multiple teeth loss, and comminuted mandibular fractures at our department. We plan to present a subsequent study regarding the use of microplate in comminuted mandibular fractures.

This study has some limitations. There were no control groups such as traditional techniques. No biomechanical stress load study was available to define the fixation points. In addition, the use of occlusal guidance would have been more helpful in the evaluation of postoperative occlusal stability. Nevertheless, we think that microplate fixation without MMF in double mandibular fractures produces satisfactory results with minimal complications and a high level of patient comfort through immediate mouth opening, good oral hygiene, and early food intake.

\section{REFERENCES}

1. Kaplan BA, Hoard MA, Park SS. Immediate mobilization following fixation of mandible fractures: a prospective, randomized study. Laryngoscope 2001;111:1520-4.

2. Dimitroulis G. Management of fractured mandibles without the use of intermaxillary wire fixation. J Oral Maxillofac Surg 2002;60:1435-8.

3. Bell RB, Wilson DM. Is the use of arch bars or interdental wire fixation necessary for successful outcomes in the open reduction and internal fixation of mandibular angle fractures? J Oral Maxillofac Surg 2008;66:2116-22

4. Fordyce AM, Lalani Z, Songra AK, Hildreth AJ, Carton AT, Hawkesford JE. Intermaxillary fixation is not usually necessary to reduce mandibular fractures. Br J Oral Maxillofac Surg 1999;37:52-7.

5. Tate GS, Ellis E 3rd, Throckmorton G. Bite forces in patients treated for mandibular angle fractures: implications for fixation recommendations. J Oral Maxillofac Surg 1994;52:734-6. 